\title{
Edge-Removal and Non-Crossing Configurations in Geometric Graphs
}

\author{
Oswin Aichholzer ${ }^{1 *}$ \\ David Flores-Peñaloza ${ }^{4}$ \\ Ferran Hurtado $6 \ddagger$
}

\author{
Sergio Cabello ${ }^{2 \dagger}$ \\ Thomas Hackl ${ }^{1 *}$ \\ Ruy Fabila-Monroy ${ }^{3}$ \\ Clemens Huemer ${ }^{5 \ddagger}$ \\ David R. Wood ${ }^{7 \S}$
}

\footnotetext{
${ }^{1}$ Institute for Software Technology, Graz University of Technology, Austria,

${ }^{2}$ Department of Mathematics, IMFM, and Department of Mathematics, FMF, University of Ljubljana, Slovenia.

${ }^{3}$ Departamento de Matemáticas, CINVESTAV, México DF, Mexico.

${ }^{4}$ Instituto de Matemáticas, Universidad Nacional Autónoma de México.

${ }^{5}$ Departament de Matemàtica Aplicada IV, Universitat Politècnica de Catalunya, Barcelona, Spain.

${ }^{6}$ Departament de Matemàtica Aplicada II, Universitat Politècnica de Catalunya, Barcelona, Spain.

${ }^{7}$ Department of Mathematics and Statistics, The University of Melbourne, Melbourne, Australia.
}

received May 19, 2008, revised November 25, 2009, accepted February 3, 2010.

A geometric graph is a graph $G=(V, E)$ drawn in the plane, such that $V$ is a point set in general position and $E$ is a set of straight-line segments whose endpoints belong to $V$. We study the following extremal problem for geometric graphs: How many arbitrary edges can be removed from a complete geometric graph with $n$ vertices such that the remaining graph still contains a certain non-crossing subgraph. The non-crossing subgraphs that we consider are perfect matchings, subtrees of a given size, and triangulations. In each case, we obtain tight bounds on the maximum number of removable edges.

Keywords: extremal graph theory, geometric graph, perfect matching, spanning tree

\footnotetext{
I A preliminary version of this paper was presented at the 24th European Workshop on Computational Geometry, 2008.

* Supported by the Austrian FWF National Research Network 'Industrial Geometry' S9205-N12.

${ }^{\dagger}$ Supported by the Slovenian Research Agency, project J1-7218.

${ }^{\ddagger}$ Supported by projects MEC MTM2006-01267, MEC MTM2009-07242 and Gen. Cat. 2005SGR00692, Gen. Cat. DGR 2009SGR1040.

$\S$ Supported by a QEII Research Fellowship from the Australian Research Council. Research completed at the Universitat Politècnica de Catalunya, where supported by the Marie Curie Fellowship 023865, and by the projects MEC MTM2006-01267 and Gen. Cat. 2005SGR00692.
}

1365-8050 @ 2010 Discrete Mathematics and Theoretical Computer Science (DMTCS), Nancy, France 


\section{Introduction}

A geometric graph is a graph $G=(V, E)$ drawn in the plane, such that $V$ is a point set in general position (meaning that no three points of $V$ lie on a common line) and $E$ is a set of straight-line segments whose endpoints belong to $V$. A convex graph is a geometric graph whose vertex set is in convex position. A geometric graph is called non-crossing if no two edges intersect in their interior, but two edges might have an endpoint in common. Two edges are disjoint if they have no point in common.

Extremal problems for geometric graphs have received considerable attention. One such problem is to determine the minimum integer $e_{k}(n)$ such that every geometric graph with $n$ vertices and $m>e_{k}(n)$ edges contains $k+1$ pairwise disjoint edges. Equivalently, $e_{k}(n)$ is the maximum number of edges in a geometric graph with $n$ vertices and no $k+1$ pairwise disjoint edges.

This problem was first studied by Erdôs [7], Perles, Kupitz, and Avital and Hanani. Accounts of the history of this topic can be found in [2, 5, 8, 19]. Erdôs [7] proved that $e_{1}(n)=n$. (This result solves Conway's thrackle conjecture for straight-line thrackles.) For three pairwise disjoint edges, bounds on $e_{2}(n)$ were given in [2, 10], culminating in $e_{2}(n)=\frac{5}{2} n$ plus a constant, as shown recently by Černý [5]. Bounds on $e_{3}(n)$ have been obtained in [10, 23].

For general values of $k$, Goddard et al. [10] showed that $e_{k}(n) \leq c n(\log n)^{k-4}$ for some constant $c$. This was improved by Pach and Törốcsik [20] to $e_{k}(n) \leq k^{4} n$, the first upper bound linear in $n$. Tóth and Valtr [23] improved the dependence on $k$ to $e_{k}(n) \leq k^{3}(n+1)$. This bound was improved (for $k \geq 2^{9}$ ) to $e_{k}(n) \leq 2^{9} k^{2} n$ by Tóth [22], where the constant $2^{9}$ has since been improved to $2^{8}$ by Felsner [8]. Kupitz proved a lower bound of $e_{k}(n)>k n$, which was improved to $e_{k}(n) \geq \frac{3}{2}(k-1) n-2 k^{2}$ by Tóth and Valtr [23]. It is conjectured that $e_{k}(n) \leq c k n$ for some constant $c$. Note that for convex graphs, this question was completely settled by Kupitz and Perles for each $k$. They showed that every convex graph with $n$ vertices and at least $k n+1$ edges contains $k+1$ pairwise disjoint edges, and this bound is tight; see [10]. Closely related work is [3, 14, 15, 16].

\subsection{Our Results}

Research on $e_{k}(n)$ has focused on small values of $k$. But $k$ can be as large as $\frac{n}{2}-1$ for even $n$, which is the case that we study. Our first contribution is to determine $e_{n-1}(2 n)$ for all $n$. By definition, $e_{n-1}(2 n)$ is the minimum integer such that every geometric graph with $2 n$ vertices and at least $e_{n-1}(2 n)+1$ edges contains a non-crossing perfect matching. Equivalently, $\left(\begin{array}{c}2 n \\ 2\end{array}\right)-\left(e_{n-1}(2 n)+1\right)$ is the maximum number of arbitrary edges that can be removed from any complete geometric graph, such that the remaining geometric graph still contains a non-crossing perfect matching. We prove that every complete geometric graph on $2 n$ vertices still contains a non-crossing perfect matching after removing any set of $n-1$ edges. That is, $\left(\begin{array}{c}2 n \\ 2\end{array}\right)-\left(e_{n-1}(2 n)+1\right) \geq n-1$, implying $e_{n-1}(2 n) \leq\left(\begin{array}{c}2 n \\ 2\end{array}\right)-n$. This bound is achieved for the complete convex graph, meaning that there exists a set of $n$ edges whose removal disallows a non-crossing perfect matching in the remaining graph. Kupitz and Perles already obtained this bound on the complete convex graph. Hence

$$
e_{n-1}(2 n)=\left(\begin{array}{c}
2 n \\
2
\end{array}\right)-n=2 n(n-1) .
$$

These results are presented in Section 2 .

Our second contribution is motivated by a closely related problem posed by Micha Perles in 2002 and studied by Černý, Dvořák, Jelínek and Kára [6]: How many edges can be removed from a complete geometric graph on $n$ vertices such that the remaining graph still contains a non-crossing Hamiltonian 
path? It is of interest to study this type of problem for other classes of non-crossing geometric graphs. We consider subtrees of a given size. For the case of spanning trees, removing $n-2$ arbitrary edges from any complete geometric graph on $n$ vertices leaves a graph that still contains a non-crossing spanning tree [13]. Removal of more edges is possible if the set of removed edges has certain properties. Benediktovich [4] recently showed that every complete geometric graph on $n \geq 5$ vertices still contains a non-crossing spanning tree after removing any self-crossing 2 -factor (that is, a 2-regular spanning subgraph with two edges sharing an interior point).

We prove that every complete geometric graph on $n$ vertices still contains a non-crossing subtree that spans $n-k$ vertices after removing $\left\lceil\frac{k n}{2}\right\rceil$ arbitrary edges, for $k \geq 2$, and this bound is tight. This result is presented in Section 3 .

Finally in Section 4, we consider the question of how many edges (not on the boundary of the convex hull) can be removed from a complete geometric graph so that the remaining geometric graph contains a triangulation or a pseudo-triangulation.

In the following, if $H$ is a subgraph of a geometric graph $G$, then $G-H$ denotes the geometric graph obtained from $G$ by deleting the edges of $H$. The edges of $H$ are called removed or forbidden edges.

\section{Perfect matchings}

In this section we determine the maximum number of arbitrary edges that can be removed from any complete geometric graph such that the remaining geometric graph still contains a non-crossing perfect matching. The proof relies on the following result for abstract graphs.

Theorem 2.1 For all $p \geq 2$, for every spanning subgraph $H=\left(V, E^{\prime}\right)$ of the complete graph $K_{k p}$ with $\left|E^{\prime}\right| \leq k-1$, the graph $K_{k p}-H$ contains the complete p-partite graph $K_{k, \ldots, k}$.

Proof: For each $p$ we prove the theorem by induction on $k$. For $k=1$ the statement is trivial. Assume the statement is true for every number $k^{\prime}<k$. Now, we are given the complete graph $K_{k p}$ and we are given a spanning subgraph $H=\left(V, E^{\prime}\right)$ with $\left|E^{\prime}\right| \leq k-1$. Assume that $\left|E^{\prime}\right|>0$, as otherwise nothing has to be proved. Observe that there exists a set $Q$ of at least $p-1$ isolated vertices in $H$ and there exists a vertex $v \notin Q$ whose degree is at least 1 in $H$. Let $N(v)$ denote the set of neighbors of $v$ in $H$. Define a graph $H^{\prime}=\left(V \backslash(Q \cup\{v\}), E^{*}\right)$ where $E^{*}$ is obtained by first taking the set of edges of the subgraph of $H$ induced by $\left(V \backslash(Q \cup\{v\}), E^{\prime}\right)$ and then adding a minimum number of edges to the resulting set, such that $N(v)$ is connected. We have $\left|E^{*}\right| \leq\left|E^{\prime}\right|-1 \leq k-2$, because we removed $\operatorname{deg}_{H}(v)$ edges and added at $\operatorname{most}_{\operatorname{deg}}(v)-1$ edges to restore the connectedness. By induction, $K_{(k-1) p}-H^{\prime}$ contains the complete $p$-partite graph $K_{k-1, \ldots, k-1}$. Since $N(v)$ is connected in $H^{\prime}$, all the vertices of $N(v)$ belong to the same vertex class of $K_{k-1, \ldots, k-1}$. Add $v$ to the vertex class containing $N(v)$, and add one vertex in $Q$ to each of the other vertex classes so that $K_{k, \ldots, k} \subseteq K_{k p}-H$.

Corollary 2.2 For every complete geometric graph $G$ on $2 n$ vertices and for every subgraph $H$ of $G$ with at most $n-1$ edges, the geometric graph $G-H$ contains a non-crossing perfect matching. This bound is tight with respect to the cardinality of the set of forbidden edges.

Proof: Apply the case $p=2$ of Theorem 2.1. which states that $G-H$ contains a complete bipartite graph $K_{n, n}$. Color the point set according to this bipartition, say red and blue. This 2-colored point set has a 

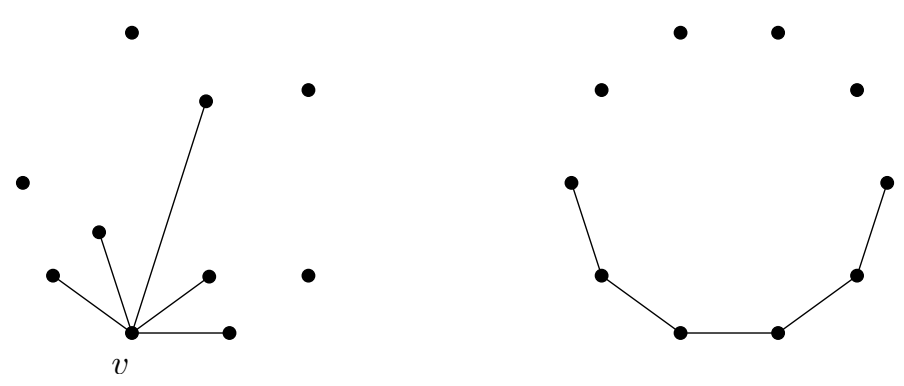

Fig. 1: Removing $n$ edges from the complete geometric graph on a set of $2 n$ points can disallow a non-crossing perfect matching.

non-crossing red-blue matching; that is, each edge of the matching connects a red and a blue point [1]. Thus, this matching does not use edges of $H$.

Removing $n$ edges from $G$ does not always leave a non-crossing perfect matching, as can be seen in Figure 1 (left). There, if the vertex $v$ is matched to another point not using the drawn 'forbidden' edges, then this segment splits the point set into two sets of odd size, which disallows a non-crossing perfect matching. Thus, the bound of $n-1$ edges is tight.

Another example that prohibits a non-crossing perfect matching without forbidden edges is shown in Figure 1 (right): Remove $n$ consecutive edges from the convex hull of the complete convex graph. Then every non-crossing perfect matching contains at least one of these $n$ forbidden edges. To see this, we use the fact that each non-crossing perfect matching on a point set in convex position has at least two edges of the convex hull [12]. Since the $n$ forbidden edges span $n+1$ points, any non-crossing perfect matching would have an edge that connects two of those $n+1$ points. However, this edge would split the point set into two smaller point sets, and in one of them all but one edge of the convex hull are forbidden, which means that it cannot have a non-crossing perfect matching. Examples for the convex geometric graph are also due to Kupitz and Perles.

In both examples the graph defined by the forbidden edges has one component that contains $n+1$ vertices. The size of the largest component in this graph turns out to be crucial for the existence of a non-crossing perfect matching without forbidden edges. To show this, we first prove a result for colored point sets (which extends a known proof for 2-colored point sets).

Theorem 2.3 Let $S$ be a set of colored points in general position in the plane with $|S|$ even. Then $S$ admits a non-crossing perfect matching such that every edge connects two points of distinct colors if and only if at most half the points in $S$ have the same color.

Proof: It is necessary for at most half the points in $S$ to have the same color, as otherwise every perfect matching would connect two points of this color. Now we prove the sufficiency. Say the colors are $1,2, \ldots, l$. Let $S_{i}$ be the set of points colored $i$, for $1 \leq i \leq \ell$. First, we construct a perfect matching without edges connecting points of the same color, but possibly with edge crossings. Thereto, choose a point from the largest color class $S_{i}$ and match it with a point from some other color class $S_{j}$. This maintains the property that every color class contains at most half the unmatched points. Continue this process until we obtain a perfect matching. (Note that Sitton [21] proved a more general result about the 


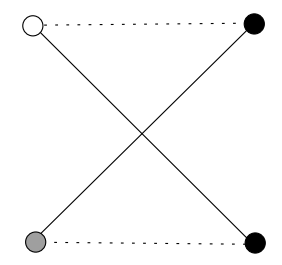

Fig. 2: Crossing removal.

size of the largest matching in an arbitrary complete multipartite graph.) Now consider a perfect matching $M$ with minimum total edge length. Suppose for the sake of contradiction that $M$ has a crossing, as illustrated in Figure 2. Observe that the corresponding 4-gon contains at least two pairwise disjoint edges connecting points of different colors (the dotted edges). By replacing the two crossing edges by these two edges of the 4-gon we obtain a perfect matching with shorter total edge length. This contradiction proves that $M$ is non-crossing.

Note that a problem related to Theorem 2.3 concerning long alternating paths for multicolored point sets was studied by Merino et al. [18].

Corollary 2.4 For every complete geometric graph $G$ on $2 n$ vertices, and for every subgraph $H$ of $G$ with at most $n$ vertices in each component, the geometric graph $G-H$ contains a non-crossing perfect matching.

Proof: Color each component with a different color and apply Theorem 2.3

Note that Corollary 2.4 also implies Corollary 2.2 .

We believe that if a complete geometric graph has many vertices in the interior of the convex hull (at least about half of the points), then more edges can be removed to still have a non-crossing perfect matching. In Figure 1 (left), $k=n-2$ points of the $2 n$ points are in the interior of the convex hull and removal of the $k+2$ solid edges disallows a non-crossing perfect matching.

Conjecture 1 For every complete geometric graph $G$ on a set of $2 n$ vertices with $k \geq n-2$ of them in the interior of the convex hull, and for every subgraph $H$ of $G$ which has at most $k+1$ edges, the geometric graph $G-H$ contains a non-crossing perfect matching.

\section{Non-crossing subtrees}

In this section we investigate how many arbitrary edges can be removed from a complete geometric graph such that the remaining graph still contains a non-crossing tree of a given size.

The case of spanning trees is a simple corollary of a theorem by Károlyi et al. [13], who proved that for every 2-coloring of the edges of a complete geometric graph $G$, at least one color class contains a noncrossing spanning tree. Thus, if we color a given set of at most $n-2$ edges red and the remaining edges blue, then the spanning tree must be blue. That is, if $n-2$ edges are removed from $G$, then the remaining graph still contains a non-crossing spanning tree. This bound is tight, since removing the $n-1$ edges incident to a single vertex disallows any spanning tree. Also, removing $n-1$ edges from the boundary of the convex hull of the complete convex graph disallows a non-crossing spanning tree. 


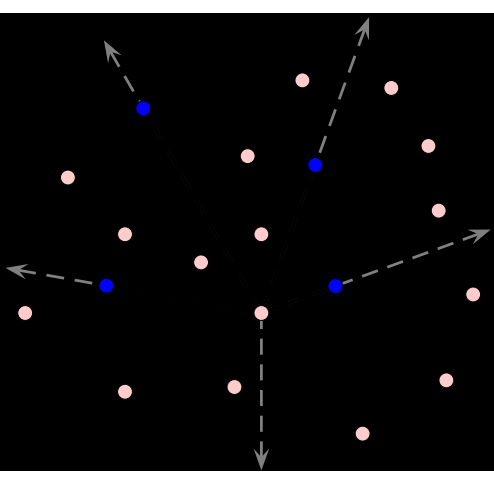

Fig. 3: Construction of $T$.

Now consider non-crossing trees that span fewer vertices. It turns out that the connectivity of the removed subgraph $H$ is crucial for the size of the largest non-crossing subtree. Recall that a graph $H$ is $k$-connected if $H$ has at least $k+1$ vertices and every cut has at least $k$ vertices, where a cut is a set of vertices whose removal disconnects $H$. The connectivity of $H$ is the maximum integer $k$ such that $H$ is $k$-connected.

Lemma 3.1 For every complete geometric graph $G$ on $n$ vertices and for every spanning subgraph $H$ of $G$ with connectivity $k$, the geometric graph $G-H$ contains a non-crossing subtree that spans $n-k$ vertices. Moreover, there is a spanning subgraph $H$ of $G$ with connectivity $k$, such that every subtree of $G-H$ has at most $n-k$ vertices.

Proof: Since $H$ is $k$-connected, it has at least $k+1$ vertices. If $H$ has exactly $k+1$ vertices, then $G-H$ contains $K_{n-k}$ as a subgraph, which contains a non-crossing spanning tree on $n-k$ vertices. Now assume that $H$ has at least $k+2$ vertices. Thus $H$ has a cut set $X$ with $k$ vertices. The graph obtained from $H$ by deleting the vertices in $X$ has at least two components. Color the vertices in one component red and color the remaining vertices (not in $X$ ) blue. Choose a red vertex $v$. We now construct a non-crossing tree $T$, as illustrated in Figure 3 First connect $v$ to every blue vertex. This defines a non-crossing star. Extend each edge of the star to an infinite ray starting at $v$. These rays divide the plane into regions. If the angle of one such region at $v$ is greater than $\pi$, then draw one auxiliary ray at $v$ in this region, so that the angle of each region at $v$ is at most $\pi$. Thus each region is convex. Each red point except $v$ lies inside one of these regions. For each region, choose a blue point on its boundary, which exists by construction. Connect in $T$ every red point in that region to the chosen blue point. Within each region $T$ is a non-crossing star. Thus $T$ is a non-crossing tree on at least $n-k$ vertices, in which each edge connects a red and a blue vertex. Hence $T$ does not use edges of $H$. To prove the final claim, let $H$ be the subgraph consisting of every edge incident to $k$ arbitrary vertices. It is easily seen that $H$ has connectivity $k$. Since $G-H$ is isomorphic to $K_{n-k}$ plus $k$ isolated vertices, every subtree of $G-H$ has at most $n-k$ vertices.

In particular, Lemma 3.1 implies that for every subgraph $H$ with $n-1$ edges of a complete geometric graph $G$ on $n$ vertices, the geometric graph $G-H$ contains a non-crossing subtree that spans $n-1$ vertices. Also, for every disconnected subgraph $H$ of a complete geometric graph $G$, the geometric graph $G-H$ contains a non-crossing spanning tree. 
For a convex graph $G$ with $n$ vertices, number the vertices $0,1, \ldots, n-1$ in counterclockwise order. The depth of an edge $i j$ is defined to be $\min \{|i-j|, n-|i-j|\}$. Edges on the boundary of the convex hull thus have depth 1. Edges with depth 2 are called ears.

Theorem 3.2 For $2 \leq k \leq n-1$, for every complete geometric graph $G$ on $n$ vertices, and for every subgraph $H$ of $G$ with at most $\lceil k n / 2\rceil-1$ edges, the geometric graph $G-H$ contains a non-crossing subtree that spans $n-k+1$ vertices. This bound is tight with respect to the cardinality of the set of forbidden edges: the complete convex graph $G$ has a subgraph $H$ with $\lceil\mathrm{kn} / 2\rceil$ edges such that every non-crossing tree in $G-H$ has at most $n-k$ vertices.

Proof: Since $H$ has at most $\lceil k n / 2\rceil-1$ edges,

$$
\sum_{v \in H} \operatorname{deg}(v) \leq 2(\lceil k n / 2\rceil-1) \leq k n-1 .
$$

Therefore, the average vertex degree is less than $k$. Consequently there exists a vertex in $H$ of degree at most $k-1$ in $H$ and thus the connectivity of $H$ is at most $k-1$. By Lemma 3.1, $G-H$ has a non-crossing subtree that spans at least $n-k+1$ vertices.

We now show that the complete convex graph $G$ with $n$ vertices has a subgraph $H$ with $\lceil k n / 2\rceil$ edges such that $G-H$ has no non-crossing tree on $n-k+1$ vertices. The subgraph $H$ is the Harary graph $H_{k, n}$ [11]. We now define this graph, and draw it as a convex graph with its vertices numbered $0,1, \ldots, n-1$ in counterclockwise order (see Figure 4). First every edge with depth at most $\lfloor k / 2\rfloor$ is in $H_{k, n}$. This adds $2\lfloor k / 2\rfloor$ edges incident to each vertex. If $k$ is odd, then in addition every edge $i j$ with $|i-j|=\lfloor n / 2\rfloor$ is in $H_{k, n}$. This adds one edge incident to each vertex, except for odd $n$, in which case the vertex $(n-1) / 2$ gets two more incident edges. It follows that $H_{k, n}$ has $\lceil k n / 2\rceil$ edges in all cases.
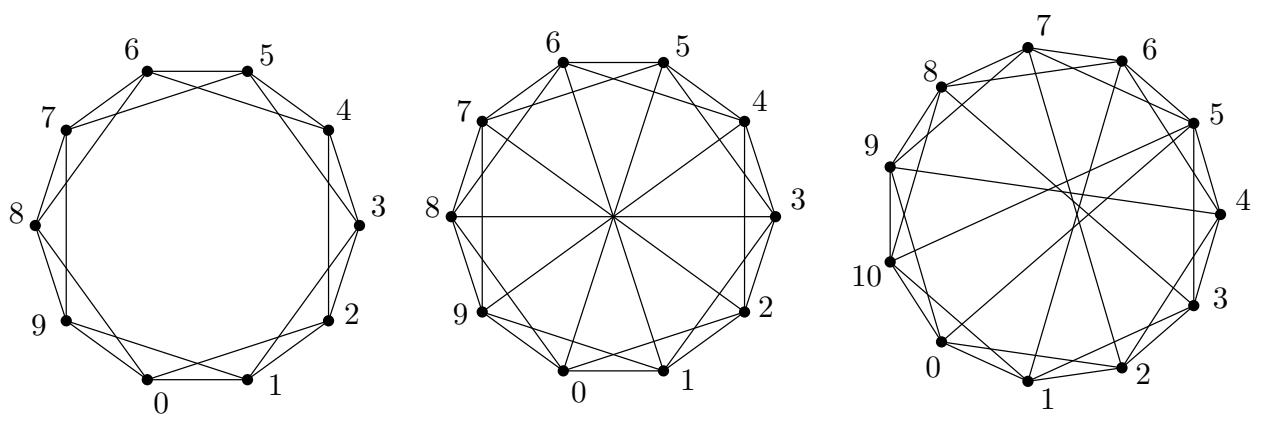

Fig. 4: The Harary graphs $H_{4,10}, H_{5,10}$ and $H_{5,11}$.

Suppose on the contrary that there is a non-crossing tree $T$ in $G-H_{k, n}$ with $t \geq n-k+1$ vertices. Consider the dual graph $D$ of $T$, which contains one vertex for each region that is bounded by edges of $T$ and the boundary of the convex hull (excluding the region outside the convex hull of $G$ ). Two vertices of $D$ are adjacent if the corresponding regions share an edge of $T$. It is well known that $D$ is a tree.

An edge of $T$ on the boundary of the convex hull of $T$ is called a boundary edge. Each boundary edge with depth $d$ forces $T$ to avoid a distinct set of $d-1$ vertices. Since $T$ avoids $H_{k, n}$, each edge in $T$ 
has depth at least $\lfloor k / 2\rfloor+1$. So if $T$ has $b$ boundary edges, then $T$ has at most $n-b\lfloor k / 2\rfloor$ vertices. Thus $n-k+1 \leq t \leq n-b\lfloor k / 2\rfloor$. García et al. [9] proved that every convex straight-line drawing of a tree has at least two boundary edges. Thus $b \geq 2$, implying $b=2, k$ is odd, and $t=n-k+1$. Moreover, García et al. [9] proved that every tree that admits a convex straight-line drawing with only two boundary edges is a caterpillar, and in this case the dual is a path. Thus $T$ is a caterpillar and $D$ is a path. As illustrated in Figure 5. T has exactly two edges $f^{\prime}$ and $f^{\prime \prime}$ of depth $(k+1) / 2$, the endpoints of $D$ correspond to the regions defined by $f^{\prime}$ and $f^{\prime \prime}$, and each such region contains $(k-1) / 2$ vertices not in $T$. Every other vertex is in $T$.
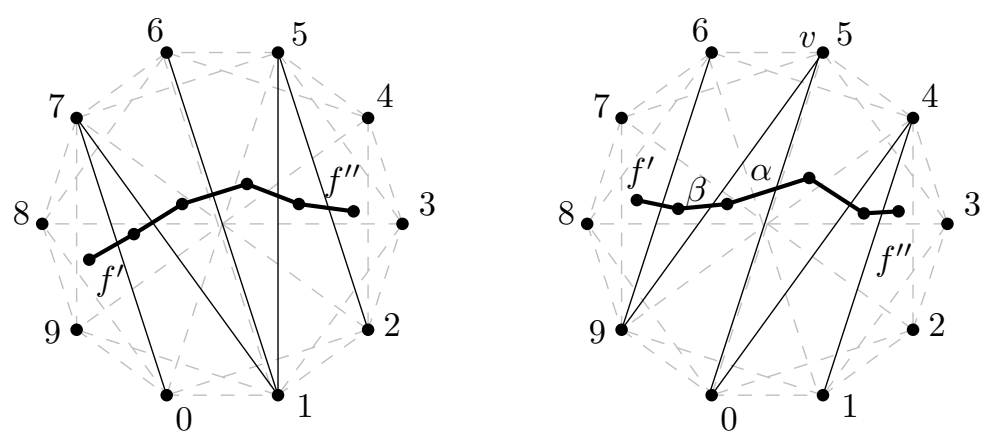

Fig. 5: The caterpillar $T$, its dual graph $D$ (bold), and the forbidden edges (dashed).

Define the function $q$ as follows. For each edge $e$ of $D$, if $i j$ is the edge of $T$ that crosses $e$, then let $q(e):=|i-j|$. Below we prove that $q(e)=\lfloor n / 2\rfloor$ for some edge $e$ of $D$. This implies that $|i-j|=\lfloor n / 2\rfloor$ for some edge $i j$ of $T$, which is a contradiction since every such edge is in $H_{k, n}$.

We now consider two cases depending on whether vertex 0 borders one of the regions defined by $f^{\prime}$ and $f^{\prime \prime}$. First suppose that 0 borders the region corresponding to $f^{\prime}$, as illustrated in Figure 5 (left). In this case, walking along $D$ starting from the edge that crosses $f^{\prime \prime}$, the function $q$ starts at $(k+1) / 2$, increases by 1 at each step, up to $n-(k+1) / 2$. Thus $q(e)=\lfloor n / 2\rfloor$ for some edge $e$ of $D$. The case in which 0 borders the region corresponding to $f^{\prime \prime}$ is analogous. Now assume that 0 borders neither region, as illustrated in Figure 5 (right). Let $v$ be the neighbor of 0 with maximum index. Thus $v$ is adjacent to $n-1$ in $T$. Let $\alpha$ be the edge of $D$ that crosses the edge $\{0, v\}$ of $T$. Let $\beta$ be the edge of $D$ that crosses the edge $\{v, n-1\}$ of $T$. First suppose that $v \geq\lfloor n / 2\rfloor$. Then walking along $D$ starting from $\alpha$ in the direction away from $\beta$, the function $q$ starts at $v$, decreases by 1 at each step, down to $(k+1) / 2$. Thus $q(e)=\lfloor n / 2\rfloor$ for some edge $e$ of $D$. Now assume that $v \leq\lfloor n / 2\rfloor-1$. Then walking along $D$ starting from $\beta$ in the direction away from $\alpha$, the function $q$ starts at $n-1-v$ (which is at least $\lfloor n / 2\rfloor$ ), then decreases by 1 at each step, down to $(k+1) / 2$. Thus $q(e)=\lfloor n / 2\rfloor$ for some edge $e$ of $D$.

\subsection{Point sets with many interior points}

We now present point sets with many interior points such that it is not possible to remove more edges than in the convex case to guarantee a non-crossing subtree of a given size in the remaining graph; cf. Theorem 3.2 with $k=2(l+1)$ and $n=h(l+1)$. 
Proposition 3.3 For all $h \geq 3$ and $l \geq 0$, there exists a point set $S$ with $h$ exterior and $\ell \cdot h$ interior points, and a subgraph $H$ of the complete geometric graph $G$ on $S$ with $(\ell+1)^{2} \cdot h$ edges such that every non-crossing subtree of the geometric graph $G-H$ spans at most $(l+1) h-2(l+1)$ vertices.

Proof: To construct $S$ take the vertices of a regular $h$-gon and place $k=\ell \cdot h$ interior points on the incircle of the $h$-gon, in such a way that the interior points are distributed in groups of equal size $\ell$. Each group of points is placed "very close" to a different edge of the $h$-gon. Within each group the distance from the points to the corresponding edge of the $h$-gon is monotonically increasing when considering the points in the counterclockwise order they are encountered along the incircle. Let $G$ be the complete geometric graph with vertex set $S$. Figure 6 (left) depicts the situation.
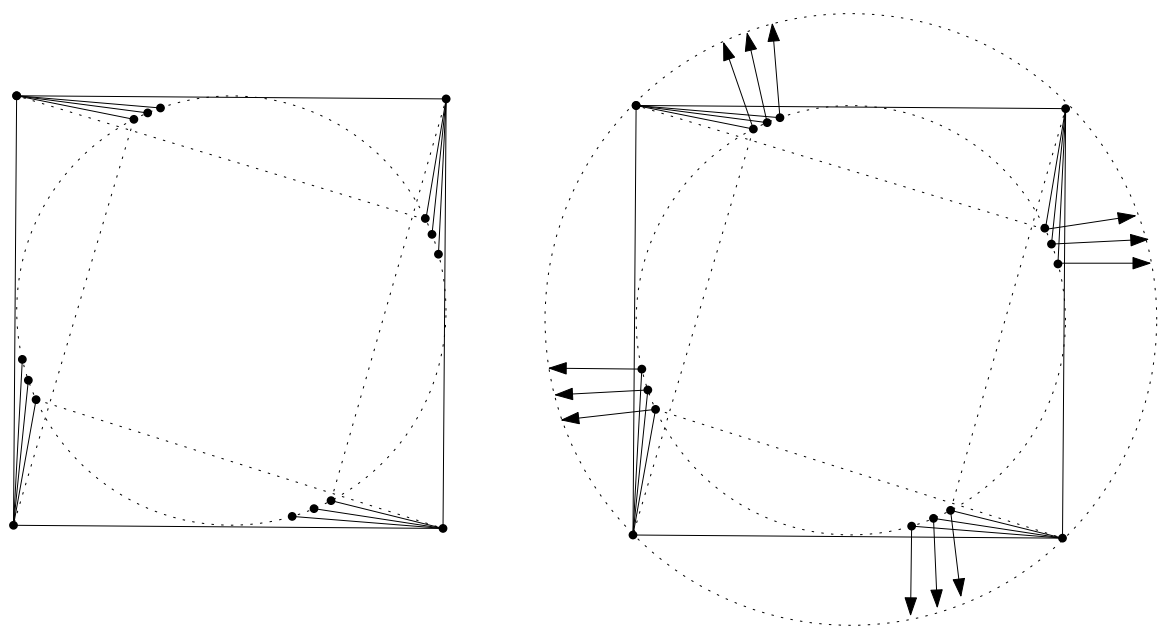

Fig. 6: Illustration for the proof of Proposition 3.3 with $h=4$ and $l=3$.

Let $S^{\prime}$ be the set of points in convex position obtained from $S$ by moving each interior point in $S$ to the circumcircle of the $h$-gon, as indicated in Figure 6 (right). Let $G^{\prime}$ be the complete geometric graph with vertex set $S^{\prime}$. Let $H$ be the Harary graph $H_{2(\ell+1),(\ell+1) \cdot h}$ defined with respect to $S^{\prime}$. Consider $H$ to also be a subgraph of $G$.

Observe that each edge-crossing produced by moving the interior points from the incircle to the circumcircle involves an edge connecting an interior point to its nearest vertex of the $h$-gon or involves an edge of the $h$-gon (the solid edges in Figure 6). Each such edge has depth at most $l+1$ in $G^{\prime}$. Each such edge is in $H$, since by the definition of the Harary graph with $k=2(l+1)$, every edge of depth at most $l+1=\lfloor k / 2\rfloor$ is in $H$. Thus every non-crossing subtree in $G-H$ is a non-crossing subtree in $G^{\prime}-H$. Since every non-crossing subtree in $G^{\prime}-H$ has at most $(l+1) h-2(l+1)$ vertices (by Theorem 3.2), every non-crossing subtree in $G-H$ has at most $(l+1) h-2(l+1)$ vertices. 


\section{Triangulations and pseudo-triangulations}

This section considers how many edges can be removed from a complete geometric graph so that the remaining geometric graph contains a triangulation or a pseudo-triangulation. In a related work, Lloyd [17] proved that it is NP-complete to decide whether a given geometric graph contains a triangulation of its vertex set.

In every complete geometric graph $G=(V, E)$, there are edges of $G$, such as those on the boundary of the convex hull, that appear in every triangulation of $V$. We call these edges unavoidable, and it is easy to see that an edge $e$ is unavoidable if and only if there exists no edge $e^{\prime}$ that crosses $e$. Every other edge is called avoidable. Since removing only one unavoidable edge disallows a triangulation, we only consider avoidable edges for removal.

First consider the convex case. Since every edge not on the boundary of the convex hull is crossed by some other edge (for $n \geq 4$ ), an edge of the complete convex graph is unavoidable if and only if it is on the boundary of the convex hull.

Theorem 4.1 For each subgraph $H=\left(V, E^{\prime}\right)$ of the complete convex graph $G$ on $n \geq 3$ vertices, where $E^{\prime}$ is a set of at most $n-3$ avoidable edges, the convex graph $G-H$ contains a triangulation. This bound is tight with respect to $\left|E^{\prime}\right|$.

Proof: We proceed by induction on the number of vertices. For $n \leq 4$ the statement is trivial. Assume the statement holds for each $n^{\prime}<n$ and that $E^{\prime} \neq \emptyset$. Recall that an ear is an edge of $G$ with depth 2 .

If no ear is in $E^{\prime}$, then let $f$ be any edge in $E^{\prime}$, and let $e$ be an ear that crosses $f$. Otherwise, some ear is in $E^{\prime}$. Since there are $n$ ears, and $\left|E^{\prime}\right|<n$, there are 'consecutive' ears $e$ and $f$ that cross, such that $e \notin E^{\prime}$ and $f \in E^{\prime}$. In both cases, there is an ear $e \notin E^{\prime}$ that crosses an edge $f \in E^{\prime}$.

Let $v$ be the vertex adjacent to both endpoints of $e$. Then $f$ is incident to $v$. So $H-v$ has at least one less forbidden edge than $H$. Thus we can apply induction with $v$ removed. We obtain a triangulation in $(G-v)-H$. Adding $e$ and the two boundary edges incident to $v$ (which are unavoidable), we obtain the desired triangulation in $G-H$.

To see that removing $n-2$ edges can disallow a triangulation, delete an ear $e$ and all $n-3$ edges that cross $e$.

If we allow interior points, then fewer edges can be removed to disallow a triangulation.

Proposition 4.2 There exist point sets with interior points, such that removing two avoidable edges disallows a triangulation.

Proof: Figure 7(left) shows an example. Observe that for every interior point of a triangulation and for every straight-line passing through this point, there must be edges incident to that point on both sides of the line. In the example, there are exactly two edges to the left of the line through the interior point. Thus at least one of these two edges is in every triangulation. And removing these two edges disallows a triangulation.

Proposition 4.2 is best possible, because by definition, every avoidable edge is in some triangulation.

We finally consider pseudo-triangulations. A pseudo-triangle is a simple polygon that has exactly three interior angles less than $\pi$. A pseudo-triangulation of a point set $S$ is a partition of the convex hull of $S$ into pseudo-triangles whose vertex set is exactly $S$. Note that for point sets in convex position, triangulations 

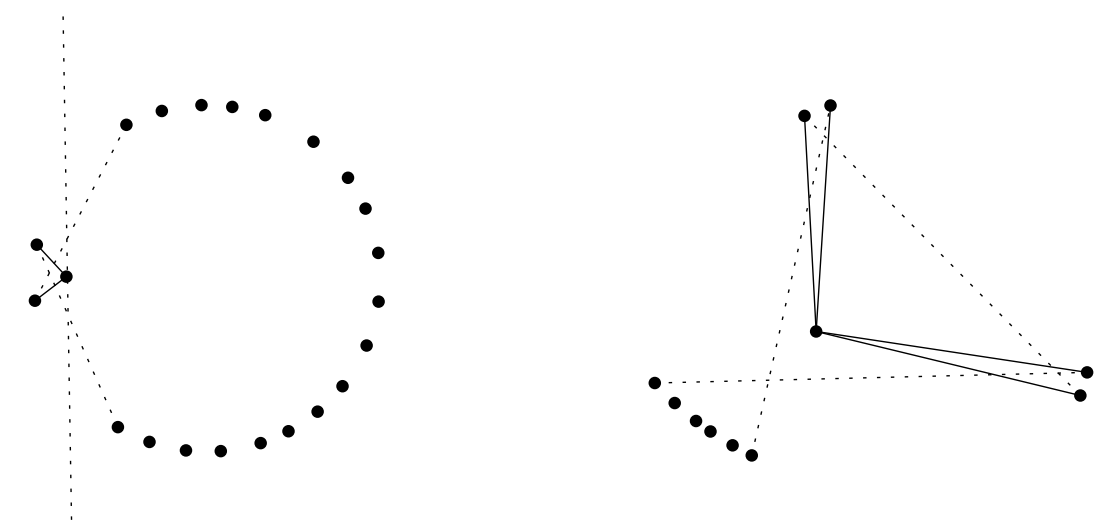

Fig. 7: Removing two avoidable edges disallows a triangulation (left), and removing four avoidable edges disallows a pseudo-triangulation (right).

and pseudo-triangulations coincide. For the question considered in Proposition 4.2, pseudo-triangulations behave similarly to triangulations.

Proposition 4.3 There exist point sets with interior points, such that removing four avoidable edges disallows a pseudo-triangulation.

Proof: Figure 7 (right) shows an example. The four solid edges are avoidable. To see that their removal disallows a pseudo-triangulation, observe that the face incident to the interior vertex with angle greater than $\pi$ has to have at least four convex vertices, whereas a pseudo-triangle has exactly three.

\section{Acknowledgements}

We thank the anonymous referees for pointing out some relevant references.

\section{References}

[1] G. L. Alexanderson, L. F. Klosinski, L. C. Larson (editors), The 1979 Putnam Exam. The William Lowell Putnam Mathematical Competition problems and solutions : 1965-1984. Mathematical Association of America, USA, 1985.

[2] N. Alon, P. Erdős, Disjoint edges in geometric graphs. Discrete and Computational Geometry 4, pp. 287-290, 1989.

[3] P. Brass, G. Károlyi, P. Valtr, A Turán-type extremal theory of convex geometric graphs. In: B. Aronov et al., editors, Discrete and Computational Geometry. The Goodman-Pollack Festschrift. Algorithms and Combinatorics 25, Berlin, Springer, pp. 275-300, 2003.

[4] V. I. Benediktovich, Plane subgraphs in geometric complement of 2-factor and complete bipartite graph. Electronic Notes in Discrete Mathematics 24, pp. 31-38, 2006.

[5] J. Černý, Geometric graphs with no three disjoint edges. Discrete and Computational Geometry 34(4), pp. 679695, 2005. 
[6] J. Černý, Z. Dvořák, V. Jelínek, J. Kára, Noncrossing Hamiltonian paths in geometric graphs. Discrete Applied Mathematics 155(9), pp. 1096-1105, 2007.

[7] P. Erdôs, On sets of distances of $n$ points. American Mathematical Monthly 53, pp. 248-250, 1946.

[8] S. Felsner, Geometric Graphs and Arrangements: Some Chapters from Combinatorial Geometry. Vieweg, Wiesbaden, 2004.

[9] A. García, C. Hernando, F. Hurtado, M. Noy, J. Tejel, Packing trees into planar graphs, Journal of Graph Theory 40(3), pp. 172-181, 2002

[10] W. Goddard, M. Katchalski, D. J. Kleitman, Forcing disjoint segments in the plane. European Journal of Combinatorics 17(4), pp. 391-395, 1996.

[11] F. Harary, The maximum connectivity of a graph. Proceedings of the National Academy of Sciences of the United States of America 48, pp. 1142-1146, 1962.

[12] C. Hernando, F. Hurtado, M. Noy, Graphs of non-crossing perfect matchings. Graphs and Combinatorics 18(3), pp. 517-532, 2002.

[13] G. Károlyi, J. Pach, G. Tóth, Ramsey-type results for geometric graphs, I. Discrete and Computational Geometry 18, pp. 247-255, 1997.

[14] G. Károlyi, J. Pach, G. Tóth, P. Valtr, Ramsey-type results for geometric graphs, II. Discrete and Computational Geometry 20, pp. 375-388, 1998.

[15] Y. S. Kupitz, On pairs of disjoint segments in convex position in the plane. In: M. Rosenfeld, J. Zaks, editors, Convexity and graph theory, Annals of Discrete Mathematics 20, pp. 203-208, 1984.

[16] Y. S. Kupitz, M. A. Perles, Extremal theory for convex matchings in convex geometric graphs. Discrete and Computational Geometry 15, pp. 195-220, 1996.

[17] E. L. Lloyd, On triangulations of a set of points in the plane. Proc. of IEEE Symposium on Foundations of Computer Science (FOCS), pp. 228-240, IEEE, 1977.

[18] C. Merino, G. Salazar, J. Urrutia, On the length of longest alternating paths for multicoloured point sets in convex position. Discrete Mathematics 306, pp. 1791-1797, 2006.

[19] J. Pach, Geometric graph theory. In: J.D. Lamb, D.A. Preece, editors, Surveys in Combinatorics, 1999. London Mathematical Society Lecture Note Series 267, Cambridge University Press, pp. 167-200, 1999.

[20] J. Pach, J. Törőcsik, Some geometric applications of Dilworth's theorem. Discrete and Computational Geometry 12, pp. 1-7, 1994.

[21] D. Sitton, Maximum matchings in complete multipartite graphs. Furman University Electronic Journal of Undergraduate Mathematics 2, pp. 6-16, 1996.

[22] G. Tóth, Note on geometric graphs. Journal of Combinatorial Theory, Series A 89(1), pp. 126-132, 2000.

[23] G. Tóth, P. Valtr, Geometric graphs with few disjoint edges. Discrete and Computational Geometry 22(4), pp. 633-642, 1999. 